\title{
К ВОПРОСУ О СЕМАНТИЧЕСКИХ ОСОБЕННОСТЯХ ОДНОРОДНЫХ КОНСТРУКЦИЙ
}

Семкина Г.Г.

Омский государственный университет им. Ф.М.Достоевского

Исследование грамматических конструкций в семантическом плане представляет собой одно из наиболее актуальных направлений современных лингвистических исследований. Семантический подход позволяет обнаружить в грамматических явлениях особенности, которые ранее не привлекали к себе внимания. Так, однородные члены предложения достаточно глубоко изучены в структурном отношении, но семантический аспект их функционирования практически не рассматривался. Само понятие однородности неоднозначно трактуется лингвистами, хотя общепринятыми можно считать три необходимых условия для идентификации элементов в качестве однородных: наличие между ними сочинительной связи; их синтаксическая связь с каким-то общим для них элементом предложения; тождество синтаксической функции. Наиболее спорным вопросом оказывается отнесение однородных конструкций к осложняющим, и, вследствие этого, структурный статус предложений с ними, который может определяться поразному в зависимости от избранного подхода.

В нашем исследовании мы поставили своей задачей обнаружить, отличаются ли однородные главные члены предложения по семантике от одноименных одиночных членов предложения. Такое сопоставление позволит выявить условия, предполагающие большую или меньшую степень вероятности функционирования определенных языковых элементов в однородном ряду, что, в свою очередь, может способствовать как уточнению отдельных лингвистических понятий, так и обоснованию общей теории осложнения предложения. В данной статье мы остановимся только на семантических особенностях однородных подлежащих.

Как известно, семантические функции подлежащего многообразны и могут быть подразделены на первичные и вторичные, в зависимости от того, совпадают ли в нем языковой и речевой компоненты значения. Соответственно, 
первичными семантическими функциями подлежащего обычно считаются функция агенса (одушевленного участника ситуации, часто человека, сознательно и целенаправленно действующего и, как правило, оказывающего какое-либо воздействие на некоторый объект с намерением достичь той или иной цели) и экспериенцера (одушевленного участника ситуации, испытывающего какую-либо эмоцию, являющегося субъектом чувственного и умственного восприятия). Во вторичных семантических функциях подлежащее обозначает другие элементы ситуации: действие или состояние, пациенс, бенефактив, объект, орудие, причину, место, время действия, элементатив. Эти данные собраны на основе исследования функционирования одиночных подлежащих. Изучение функционирования рядов однородных подлежащих выдвигает на первый план еще одну семантическую функцию, которая обычно даже не упоминается в связи с одиночными подлежащими: функцию обозначения лиц или предметов, бытие, отсутствие, наличие которых раскрывается в сказуемом. По нашим данным, около половины однородных рядов подлежащих передают именно такую семантику в ситуациях описания обстановки или перечисления участников событий в так называемых бытийных (экзистенциальных) предложениях, которые в английском языке могут быть представлены как с помощью конструкции с there, так и без нее (мы рассматриваем экзистенциальные предикаты широко, т.е. включаем в исследование не только примеры с глаголами to be, to exsist, которые передают экзистенцию в чистом виде, но и с глаголами to stand, to hang, to lie, to appear и др., содержащими добавочное сообщение о форме или моменте существования). Таким образом, можно предположить, что, с одной стороны, значение бытийности является характерной семантической особенностью однородных рядов подлежащих, и, с другой стороны, для бытийных предложений наличие однородных подлежащих достаточно частотно. Приведем примеры: 
There was a library, and a huge kitchen with an iron stove and a large pine worktable and off of that was a butler's pantry and laundry room [Dreiser 2004: 206].

The heavy mahogany table and sideboards, the massive silver, the bright rag rugs on the shining floor were all in their accustomed places... [Mitchell 2004: 58].

As he came toward her, she saw that there was grief in his eyes and also dislike and contempt that flooded her frightened heart with guilt [Mitchell 2004: 169].

Rich and poor, black and white, women and children, the old, the dying, the crippled, the wounded... crowded the road to Atlanta...[Mitchell 2004: 5].

Как можно увидеть, субъектами бытия оказываются как одушевленные, так и неодушевленные, как конкретные, так и абстрактные предметные элементы действительности, представленные в виде совокупности.

Вторая по частотности семантическая группа однородных подлежащных рядов представлена агенсами при процессных акциональных сказуемых, причем обычно в этом случае однородные подлежащие обозначают несколько тесно связанных между собой людей, занимающихся совместной деятельностью, поэтому по способу выражения среди агенсов преобладают собственные существительные, нарицательные наименования родственников и близких людей, а также личные местоимения, например:

He and his wife had taken their intended drive, and were returning home [Austen 1994: 89].

Martha and her husband had gone to Boston [Dreiser 2004: 290].

Кроме того, однородные подлежащие могут передавать семантику экспериенцера, хотя ситуации, когда несколько лиц испытывают одновременно одно и то же состояние, выраженное статальным сказуемым, являются менее частотными. Приведем несколько примеров:

Ashley and the girls'll be glad to have us for supper [Mitchell 2004: 19].

So, James and Andrew were as startled as anyone when the news came out...[ Austen 1994: 52]. 
It cannot be doubted that Sir Walter and Elizabeth were shocked and mortified by the loss of their companion...[ Austen 1994: 253].

Однородные ряды подлежащих употребляются и для обозначения группы носителей одной и той же характеристики при именном квалификативном предикате, обычно выраженном прилагательным, передающим эту характеристику. Отметим, что в этой семантической роли преимущественно выступают неодушевленные существительные, например:

The gardens and shrubberies would be kept in almost as high order as they are now [Austen 1994: 16].

The room and the house were silent...[ Austen 1994: 188].

Подводя итог, можно отметить, сто семантика однородных рядов подлежащих в значительной степени взаимосвязана с общей семантикой ситуации: поскольку далеко не все предикаты предполагают больше одного непосредственно вовлеченного участника, постольку многие из них практически не функционируют при однородных рядах подлежащих. В частности, в однородных рядах встречаются лишь единичные подлежащиепациенсы, бенефактивы, объекты, локативы, и практически отсутствуют подлежащие-инструментативы, причины, элементативы. Таким образом, семантический состав однородных подлежащих значительно более ограничен по сравнению с одиночными подлежащими, их характерной особенностью можно считать высокую степень употребления в бытийных предложения; достаточно распространенными являются ситуации, в которых подлежащие обозначают группу участников-агенсов или экспериенцеров, а также группу объектов характеризации. Важно, что во всех этих случаях однородные подлежащие представляют единую предметную совокупность, сплоченную каким-либо объединяющим фактором: единством пространства, близкими или родственными отношениями, качественной общностью. Именно поэтому предложения с однородными подлежащими не могут быть трансформированы в ряд простых или в сложное предложение: такое преобразование было бы коммуникативно необоснованным и структурно избыточным. С другой 
стороны, единство предметного элемента сближает обсуждаемые предложения с простыми. Вышеназванные семантические особенности свидетельствуют о необходимости включения предложений с однородными подлежащими в ряд структурно и семантически осложненных, причем в континууме осложненных предложений они будут расположены ближе к полюсу простых предложений.

\section{Библиографический список}

1. Austen J. Persuasion. Penguin books., 1994.

2. Dreiser Th. Jennie Gerhardt. M., 2004.

3. Mitchell M. Gone with the Wind. M., 2004. 